\title{
Télémesure hydrologique \\ par réseau téléphonique commuté
}

\author{
Hydrological measurement \\ using a public telephone system
}

E. Persoons

Professeur au Département de Génie Rural de l'Université Catholique de Louvain, Belgique

\section{J. Laurent}

Ingénieur Principal des Ponts et Chaussées, Chef de Service au Service d'Etudes Hydrologiques du Ministère des Travaux Publics, Belgique

\section{Description du système}

\section{Principe général}

Le système est basé sur une transmission de renseignements par ligne téléphonique commutée du réseau public.

A l'endroit de prise de renseignements est installé le répondeur automatique qui, sollicité par un appel téléphonique, se connecte à la ligne et émet une fré- quence ou une série de fréquences fonction de la position du ou des capteurs auxquels il est relié. La figure 1 représente un réseau composé de capteurs de niveaux d'eau, de pluie, de vitesse de vent, de trafic routier.

A l'autre bout de la ligne, passant par les différents centraux téléphoniques, est installé le poste-chef et son système d'enregistrement. Ce dernier possède une mémoire de plusieurs centaines de numéros de téléphone. Il appelle périodiquement chaque répondeur automatique à des intervalles préétablis variables de une fois l'heure à une fois la semaine.

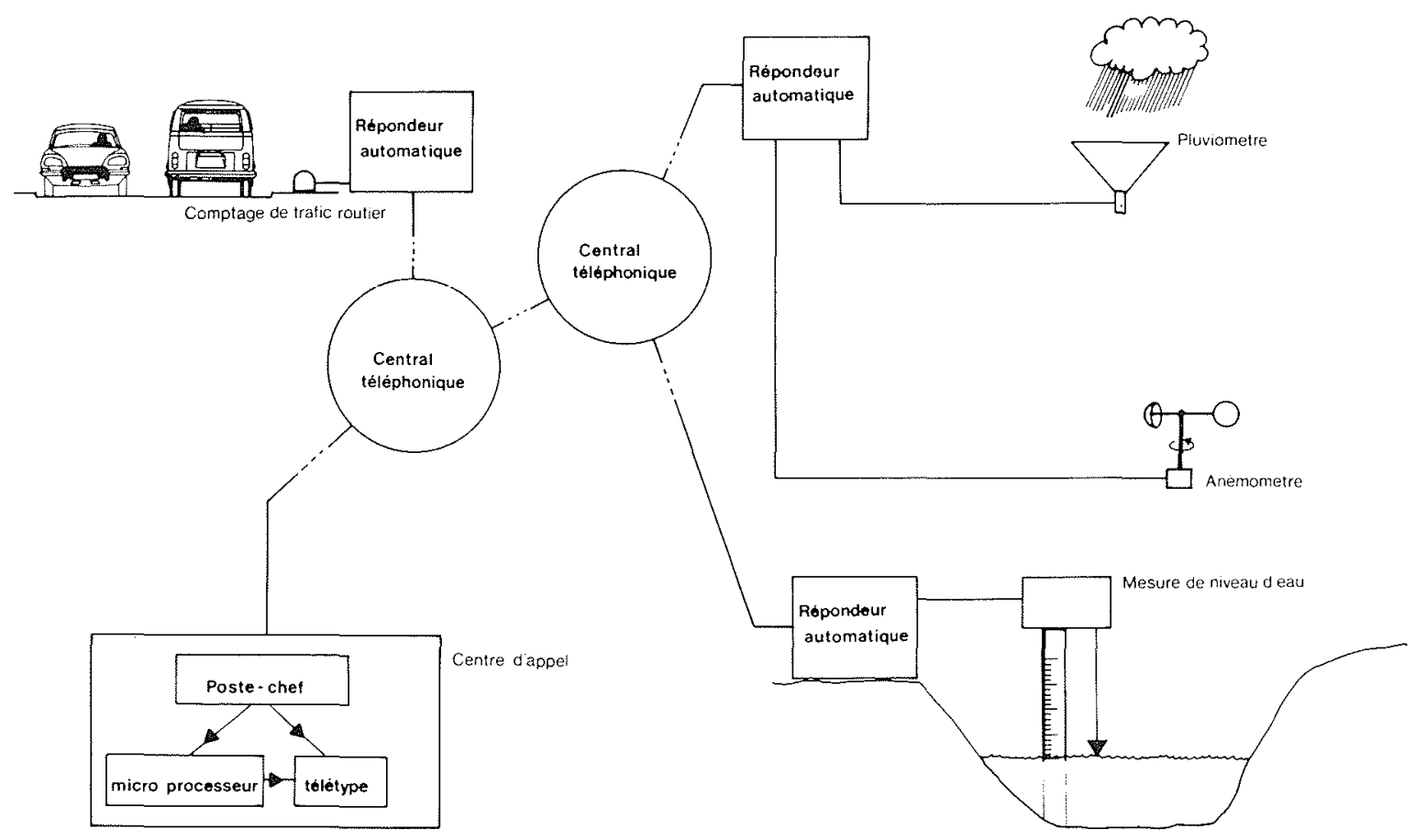

Figure 1. - Réseau type.

LA HOUILLE BLANCHE/N ${ }^{\circ} 7 / 8-1981$ 
Les fréquences émises par le répondeur sont mesurées par le fréquencemètre à sortie digitale du postechef. Cette information est, soit enregistrée sur un support informatique, un ruban papier d'une télétype, soit transmise vers un système intelligent ordinateur ou micro-processeur, afin d'obtenir un traitement immédiat.

\section{Répondeur automatique}

Sur le terrain, à l'endroit de prise d'informations se trouvent les répondeurs automatiques reliés à la ligne téléphonique. Ces répondeurs comprennent :

\section{Le décrocheur-raccrocheur (Fig. 2) composé}

- d'un détecteur du courant de sonnerie qui commande le décrochage de l'appareil ;

- d'un séquenceur de transmission qui donne la transmission des mesures, commande la liaison vers les différents capteurs et le raccrochage en fin de transmission ;

- d'un multivibrateur qui transforme le signal mesuré en une fréquence. Ce multivibrateur est relié par des relais et des interfaces vers les différents capteurs.

\section{Les interfaces vers les capteurs}

Ces interfaces sont de deux types:

\section{Répondeurs à mesures instantanées}

Ces répondeurs transmettent sur le réseau téléphonique, lors d'un appel, une information correspondant à la valeur au moment de la transmission. Dans ce cas, l'interface est composé de relais de commutation et de l'électronique ajustant la compatibilité du capteur au signal demandé par le multivibrateur. Ce type de répondeur n'est en fonctionnement qu'au moment de l'appel, il ne nécessite aucune alimentation extérieure et peut donc être relié directement à la ligne sans aucune énergie complémentaire à celle du réseau téléphonique.

\section{Répondeur à mémorisation}

Dans de nombreuses applications, telle celle de la mesure de la pluie, il est indispensable de disposer localement d'une mémoire permettant l'enregistrement du phénomène sur place entre les appels téléphoniques. Les répondeurs à mémoires locales remplissent cette fonction, c'est-à-dire qu'entre le décrocheur-raccrocheur et le capteur est intercalée une mémoire qui enregistre le phénomène lorsqu'il se produit et vide sa mémoire sur la ligne téléphonique à la demande.

\section{Exemples de répondeurs}

\section{Mesure instantanée de niveaux d'eau}

Les répondeurs à mesures instantanées fonctionnent de la manière suivante. Lors d'un appel téléphonique (Fig. 3), le détecteur de courant de sonnerie relie le multivibrateur (VCO) à la ligne téléphonique. La commande du VCO se fait par l'intermédiaire de relais alimentés à partir d'un séquenceur qui commute successivement le point haut, le point bas et le curseur d'un

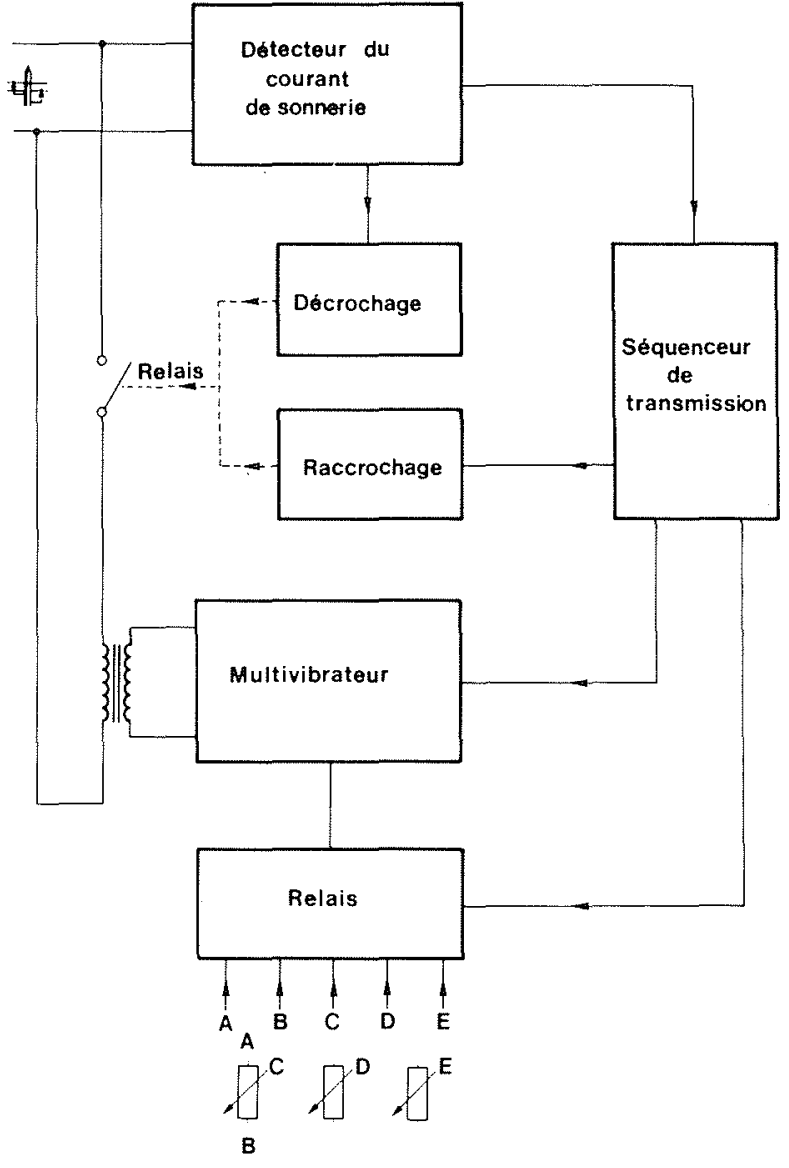

Figure 2. -- Décrocheur-raccrocheur.

potentiomètre rotatif 10 tours. Ce potentiomètre est en fait le capteur car sa rotation commandée par un câble, une poulie et un flotteur lie la variation de hauteur d'eau à une variation de résistance électrique. Celle-ci est utilisée en pont diviseur. De ce fait, la variation de hauteur d'eau entraine une variation de la tension d'alimentation du multivibrateur et donc de sa fréquence. C'est la mesure de cette fréquence qui permet de connaître à distance la hauteur d'eau.

A chaque appel on mesure la fréquence haute : $f_{H}$, la fréquence basse $f_{B}$ et la fréquence de mesure $f_{M}$.

La position $(P)$ du potentiomètre est donnée par :

$$
P=\frac{f_{M}-f_{B}}{f_{H}-f_{B}} \text { avec } 0<P<1
$$

La hauteur d'eau, quant à elle, est calculée à partir de $: H=A . P+B$. Avec

A coefficient fonction du rayon de la poulie et du potentiomètre

B coefficient fonction du positionnement du flotteur par rapport à l'échelle limnimétrique de référence.

La position du potentiomètre est déterminée à moins de $0,1 \%$. Il est certain que l'erreur due au flotteur ne nous permet pas d'attendre une restitution de la hauteur d'eau à $0,1 \%$. En pratique, en combinant toutes les erreurs tant mécaniques qu'électroniques, la télétransmission permet de l'ajuster à 0,2 à $0,4 \%$. 


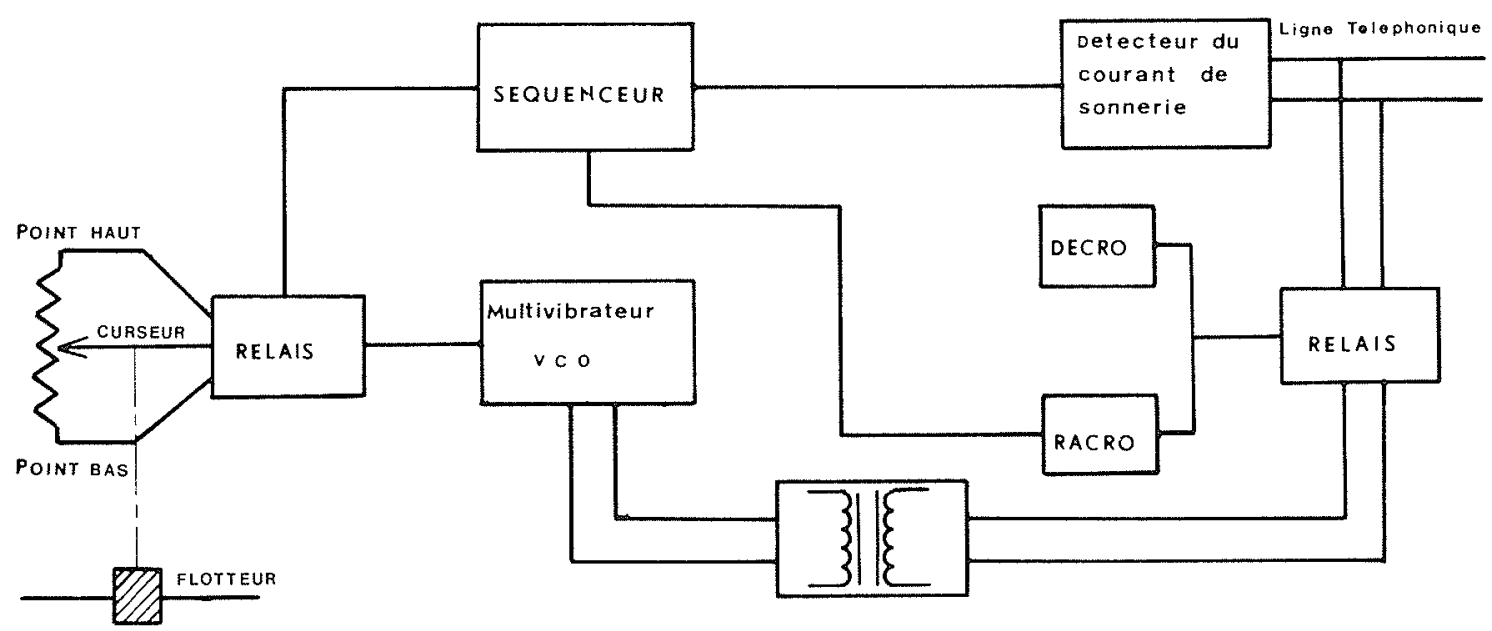

Figure 3. - Télémesure de hauteur d'eau.

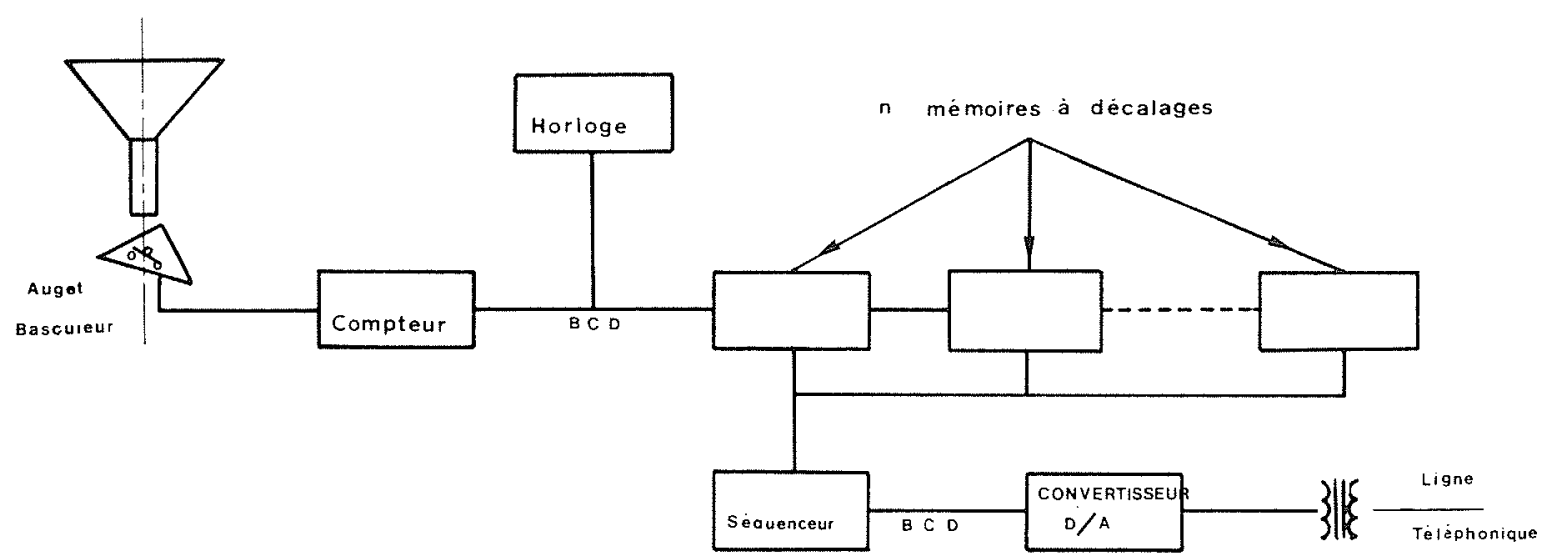

Figure 4. - Télémesure de pluie.

Les fréquences $600-1800 \mathrm{~Hz}$ ont été choisies pour rester dans le domaine des fréquences agréées au niveau des réseaux téléphoniques. Le séquenceur permet de se commuter sur plusieurs points de mesures du type potentiomètre, $t^{\circ}$, etc., et tout signal $0-20 \mathrm{~mA}$ ou 0-100 mV.

\section{Mesures mémorisées de pluie}

La mesure de la pluie nécessite une mémoire locale permettant d'enregistrer le phénomène entre les appels téléphoniques.

Le principe utilisé est le suivant. L'instrument de mesure est le pluviographe à augets basculeurs. Un dispositif électrique transforme le basculement en une impulsion électrique, laquelle est comptabilisée dans un compteur électronique en information binaire (Fig. 4). Sur l'interface électronique se trouve une horloge qui envoie le contenu du compteur dans une mé. moire à décalage en $\mathrm{BCD}$. A chaque impulsion de l'horloge (15 min ou $1 \mathrm{~h}$ ), l'état des mémoires est décalé dans 10 ou 20 mémoires, soit une durée de mémorisation de $10 \mathrm{~h}(10 \times 1 \mathrm{~h})$ ou $5 \mathrm{~h}(20 \times 15 \mathrm{~min})$. L'état des compteurs est vidé sur la ligne téléphonique lors d'appels qui se font toutes les $8 \mathrm{~h}$ ( 3 fois par jour) ou toutes les $4 \mathrm{~h}$ ( 6 fois par jour).

La difficulté consiste à transmettre l'état des mémoires en $\mathrm{BCD}$ sur la ligne téléphonique en analogique pure afin d'éliminer la présence de MODEM exigé par les services téléphoniques car ces appareils sont d'une part coûteux, d'autre part exigent une puissance électrique nécessitant le secteur.

Un convertisseur digital-fréquence transforme le nombre binaire stocké dans les mémoires à décalage en un signal analogique (fréquence) qui est transmis à l'interface de ligne. Les nombres sont représentés en 9 bits et leur valeur est comprise entre 0 et 511 .

L'exemple de la figure 5 permet de se rendre compte du message tel que le reçoit l'utilisateur.

\section{Poste-chef}

La version actuelle du poste-chef est construite autour d'un microprocesseur MOTOROLA 6800.

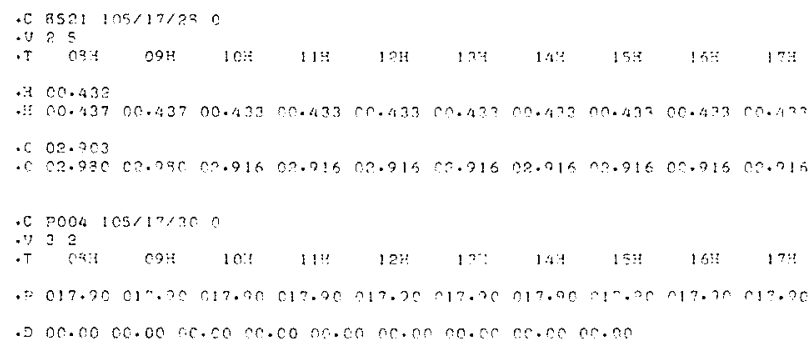

Figure 5. - Message de hauteur/débit et pluie. 
Les fonctions du poste sont :

- émission des numéros de téléphone;

- établissement de la liaison avec les répondeurs ;

- mesure des fréquences reçues;

- décodage et transformation des signaux en mesures directement utilisables ;

- transmission vers les périphériques ou vers des mémoires de masse.

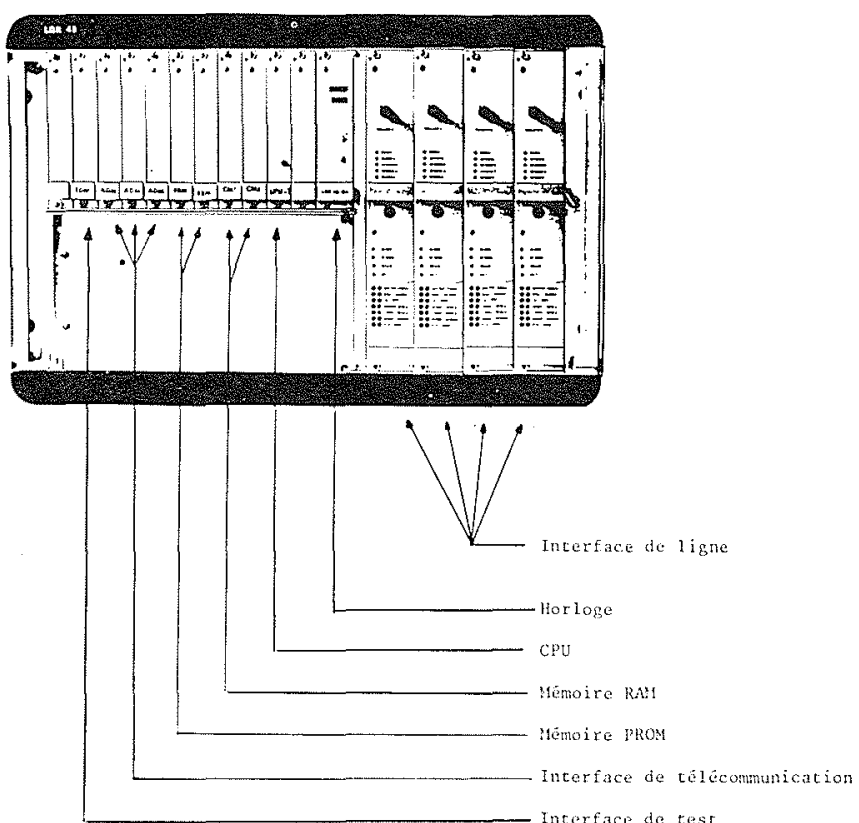

Figure 6. - Poste-chef.

Elles sont réalisées dans un poste-chef tel que celui représenté à la figure 6 . Il comporte :

- 1 à 4 interfaces pour lignes téléphoniques ;

- 1 horloge fournissant le jour, l'heure et la minute ;

- le CPU;

- les mémoires PROM ;

- les mémoires RAM;

- les interfaces asynchrones.

Le logiciel comporte de 8000 à 12000 instructions.

L'organisation des mémoires est la suivante:

- en PROM (Programmable Read Only Memory),

- le programme de base ;

- les numéros de téléphone ;

- les coefficients fixes.

- en RAM (Random Memory),

- la gestion des appels ;

- les coefficients variables (par exemple : les coefficients a et $b$ de passage de la fréquence à la hauteur d'eau).

Les appels se font soit à la demande par instruction de type CALL 8.521, soit suivant une séquence prédéterminée. Un jeu d'instructions permet de programmer à la demande les séquences d'appel.

\section{Application du système}

\section{Description du réseau}

Le système tel que décrit précédemment est utilisé dans quatre réseaux de Belgique :

- Service d'Etudes Hydrologiques du Ministère des Travaux Publics ;

- Noyau de l'Eau du Ministère de la Santé Publique et de l'Environnement ;

- Régie des Voies Aériennes;

- Service du Trafic Routier.

Huit postes d'appel à microprocesseur fonctionnent en opérationnel sur ces réseaux. Plus de deux cent postes de terrain existent et cent cinquante seront installés prochainement.

Au niveau hydrologique, nous retiendrons tout particulièrement, le réseau du Service d'Etudes Hydrologiques (SETHY). Au départ, il a été implanté pour accélérer la collecte des mesures hydrologiques. Actuellement, il sert à la statistique et au temps réel.

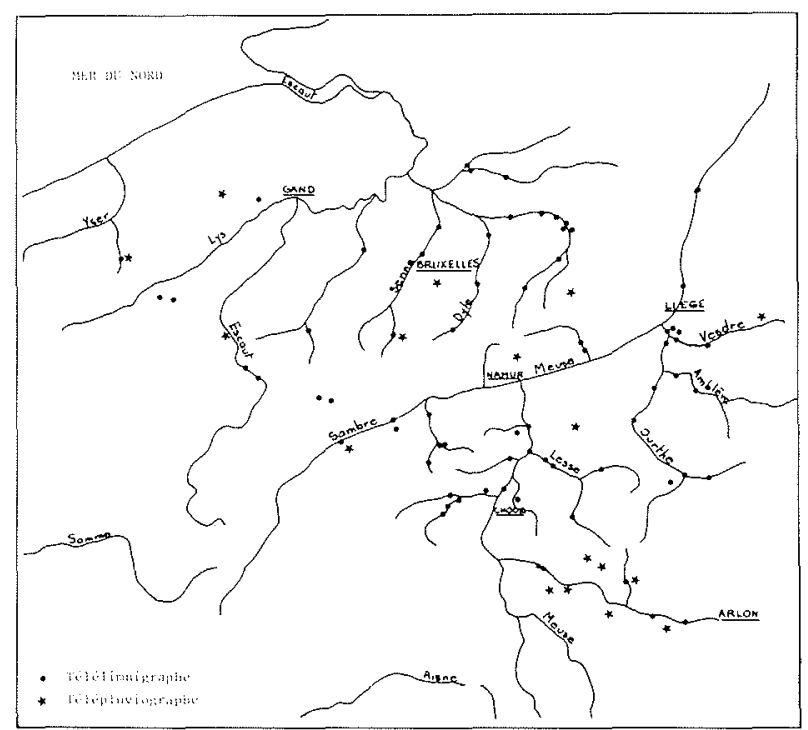

Figure 7. - Réseau actuel LGR. Mars 1981.

La carte de Belgique (Fig. 7) donne la situation actuelle de ce réseau qui comprend 65 télélimnimètres et 15 télépluviomètres. La télémesure des hauteurs d'eau se fait grâce à des limnigraphes classiques type Neyrtec, Ott ou Rittmeyer équipés d'un potentiomètre 10 tours tel que décrit dans la première partie. Les pluviographes sont des Précis Mécanique équipés d'un détecteur de basculement.

\section{Une crue exceptionnelle}

Les 20 et 21 juillet 1980 s'est produit, dans le bassin de la Meuse de la Belgique un épisode pluvieux particulièrement important qui a entrainé des crues extra- 
ordinaires sur la Meuse et ses affluents de la région Liégeoise. Les faits :

\section{La pluie}

Pour ces deux jours, la pluie totale moyenne est estimée à :

- $70 \mathrm{~mm}$ sur $64 \mathrm{~h}$, période de retour $1 / 35$ ans ;

- $60 \mathrm{~mm}$ sur $48 \mathrm{~h}$, période de retour $1 / 22$ ans;

- $40 \mathrm{~mm}$ sur $24 \mathrm{~h}$, période de retour $1 / 5$ ans.

Avec des maxima à Hastière :

- $87,9 \mathrm{~mm}$ sur $64 \mathrm{~h}$, période de retour plus de $1 / 100$ ans ;

- 82,1 mm sur $48 \mathrm{~h}$, période de retour plus de $1 / 100$ ans ;

- 48,8 $\mathrm{mm}$ sur $24 \mathrm{~h}$, période de retour $1 / 12$ ans; soit des épisodes pluvieux qui bien qu'élevés ne sont pas catastrophiques en ce qui concerne la moyenne sur le bassin.

\section{Etat hydrique du sol}

En fait, le ruissellement des sols fut nettement plus important que normalement par le fait que le mois de juillet 1980 fut particulièrement pluvieux, plus de $200 \mathrm{~mm}$ pour le mois, contre $74 \mathrm{~mm} /$ moyenne 1901. 1930.

Le total des précipitations de juin et des 20 premiers jours de juillet est de $266,2 \mathrm{~mm}$, ce qui n'a été dépassé qu'une seule fois depuis 1833 (en 1888 : $P=287,4 \mathrm{~mm}$ ).

Les sols étaient donc gorgés d'eau et la partie de la pluie qui doit normalement être retenue en détention superficielle fut négligeable.

\section{Les débits}

- Meuse à Ampsin-Neuville $\left(16400 \mathrm{~km}^{2}\right)$ avec un débit maximum de $1900 \mathrm{~m}^{3} / \mathrm{s}$. Cette crue est exceptionnelle pour l'été, mais comparable à plusieurs grandes crues des 50 dernières années en hiver.

- Meuse à Liège $\left(20100 \mathrm{~km}^{2}\right)$ avec un débit maximum de $2500 \mathrm{~m}^{3} / \mathrm{s}$.

Ces débits sont exceptionnels en été (nous n'en connaissons pas d'autres exemples) mais assez fréquents en hiver ( 5 en 50 ans).

\section{Le temps réel}

La disponibilité de mesure en temps réel a permis de donner des instructions aux gestionnaires des réseaux hydrologiques, mais aurait pu limiter considérablement les dégâts si un modèle d'interprétation des données avait été opérationnel.

L'analyse a posteriori des mesures télépluviographiques montre clairement que, dès le 20 juillet vers $14 \mathrm{~h}$, la Lesse, un des affluents importants de la Meuse, allait avoir une crue importante et, à $12 \mathrm{~h}$ il était certain qu'elle serait catastrophique.

\section{Conclusion}

Le système de télémesure utilisant le réseau téléphonique commuté pour la télétransmission permet à ce jour d'entrevoir des possibilités réelles de gestion en temps réel en hydrologie.

Lors de la crue importante des 20 et 21 juillet 1980 sur la Meuse, les performances du système ont pu être éprouvées. Il en ressort que le système est opérationnel et qu'il est indispensable à ce jour de mettre en œuvre des dispositifs intelligents permettant de fournir automatiquement les instructions nécessaires.

\section{Bibliographie}

LAURENT J., HEYNDERICKX J., DE WORM Y., et DE HOE J.M. - Le 21 juillet 1980, une crue cent cinquantenaire. Acta hydrologica Belgica, Ministère des Travaux Public, Belgique, 1980.

HEYLEN J. et LAURENT J. - De hoge waterstanden van 14-18 junuari 1981. Acta Hydrologica Belgica, Ministère des Travaux Publics, Belgique, 1981.

GASPARD S. - Evaluation des pluies exceptionnelles en Belgique. Centre Belge d'Etudes et de Documentation des Eaux, 1974, n 365 .

D'HAENE P. - Données pluviométriques pour le calcul des égouts. Centre Belge d'Etudes et de Documentation des Eaux, 1974, n 365 .

PERSOONS E., BAZIER G., ROUVET J.C. and MUSCH S. Application of telemeasurement by public telephone network to river modeling and river flow forecasting. World Meteorological Organization, Geneva, Seminar on Modern Developments in Hydrometry, Padoue, Italy, WMO, 1975, n 427 pp. 461-475.

PERSOONS E., BAZIER G., MUSCH S. and ROUVET J.C. A telemetry system working through the public telephone network. Symposium on Weather Radar and Water Management, Chester, England, 1975, 11 p., Paper 11.

BAZIER G., GEVERS M. and PERSOONS E. - An approach to follow the evolution of the base flow of sinall watersheds. Modeling and Simulation of Land, Air and Water Resources Systems, IFIP, Working Conference, Ghent, 1977, $10 \mathrm{p}$. 2 figures. 


\section{Discussion}

Président : M. P. BROCHET

Le Président. - Je remercie M. PERSOONS de son exposé. J'ai tout d'abord retenu de ce réseau son aspect pluri-disciplinaire. Par ailleurs, le procédé que vous avez développé semble très séduisant par l'économie qu'il fait d'une interface coûteuse nécessaire à tout vecteur de transmission. Enfin, ce réseau n'est pas seulement une expérience, mais un système opérationnel, et à ce titre constitue un exemple important.

M. GUILLOT. - Pour transmettre une valeur, quel est le temps d'émission de la fréquence que vous utilisez?

M. PERSOONS. - Nous avons d'abord travaillé avec une mesure de la fréquence de 6 secondes. Puis nous sommes descendus à 1 seconde, ce qui nous permet de restituer les nombres de 0 à 512 au digit exact, avec une erreur pour 500 cas. C'est acceptable. Le développement des techniques électroniques permet un réajustement très précis à partir de la fréquence précédente (en une seconde, vous pouvez mesurer à $0,1 \mathrm{~Hz}$ près).

Nous cherchons à passer à 50 millisecondes, mais n'en sommes encore qu'au prototype de laboratoire, et nous comptons un an de mise au point avant d'utiliser un tel matériel sur le terrain.

M. P.A. ROCHE. - Quels taux de pannes avez-vous sur le réseau en fonctionnement?

M. PERSOONS. - Le Service d'Etudes Hydrologiques a publié un rapport sur la crue du 21 juillet, 10 jours après celle-ci. Il y est indiqué que le taux de réponses valables a été de $92 \%$ au niveau de l'u tilisateur.

M. BEDIOT. - En France, de nombreux réseaux de télétransmission sont installés (Gard, Garonne, Py rénées Orientales) ou en cours d'installation on en projet (Loire, Seine). En ce qui concerne le réseau Seine, nous expérimentons avec nos collègues du S.R.A.E. Ile de France, la transmission par le système ARGOS des hauteurs d'un limnigraphe (Saint Cyr Sous Dourdan). En mémorisant l'information pendant 6 heures sur le site, on obtiendra des transmissions redondantes toutes les 3 heures environ, lors du passage du satellite. Mais ceci n'est qu'un aspect du réseau.

Nous envisageons également la télétransmission de données de pluic au pas de temps de la minute pour les besoins de l'hy drologie urbaine. M. PERSOONS, pouvez-vous nous indiquer pourquoi vous transmettez la pluie à un pas de temps d'un quart d'heure?

M. PERSOONS. - Le nombre d'informations que vous envisagez de transmettre n'est certainement pas compatible avec la transmission dont j'ai parlé. Il est nécessaire de digitaliser les informations, et le système est lourd et coûteux.

Néanmoins, un système de télétransmission permet effectivement de valoriser des informations au pas de la minute, dans la mesure où il résout le problème de la synchronisation. Peutêtre existe-t-il en France des solutions plus simples pour la synchronisation (horloge pilotée par une porteuse France Inter par exemple).

Mais le réseau auto-commuté, tel que nous l'utilisons présente plusicurs autres avantages : on évite l'essentiel des ruptures de cables, et on n'a pas besoin d'alimentation (tout ce qu'il faut, c'est une ligne téléphonique).

Les ruptures de cables ont été la cause de très faibles taux de fiabilité (30 à $40 \%)$ pour un réseau qui avait été installé sous les voies navigables en Belgique. Comme, de plus, nous avons obtenu la possibilité de raccorder les postes d'appel à plusieurs centraux téléphoniques, nous avons par le réseau auto-commuté amélioré considérablement cette fiabilité.

M. GUILLOT. - Des modems en circuits intégrés, coûtant quelques dizaines de francs et consommant très peu, peuvent être maintenant utilisés pour la transmission en digital. Les 2 techniques de transmission : "analogique par fréquence musicale" et "digitale", sont à mettre en concurrence, pour arriver à un progrès décisif du rapport performances/coût.
M. P.A. ROCHE. - Peut-on avoir des détails sur ces modems à 10 francs? Pour le réseau Seine, nous étions quant à nous prêts à mettre beaucoup plus que cela dans un modem.

M. PERSOONS. -- Une chose est d'avoir un circuit intégré, une autre est de le monter sur l'interface, et c'est cela qui coûte cher.

M. GUILLOT. - J'adhère pleinement aux idées du $\mathrm{Dr}$ PERSOONS sur les avantages techniques du réseau téléphonique public auto-commuté (réseau maillé, coût minimum, etc.). J'y vois une autre catégorie d'avantages déontologiques : il est navrant et rétrograde que chaque secteur d'activité veuille avoir son réseau spécialisé de transmission, il vaut bien mieux pour l'in térêt général qu'il y ait un réseau public fiable, et que tout le monde l'utilise. Le réseau public français a beaucoup progressé ces dernières années, il sort du stade de sous-développement. Autre avantage fondamental : le réseau public permet l'accès multiple à la source de l'information hydrologique, ce qui est un double facteur de progrès : cela interdit la rétention, voire la déformation des données, par un monopole quel qu'il soit ; cela crée une exigence de qualité.

M. P.A. ROCHE - Les spécialistes de la SOFRECOM et des PTT qui participent à notre groupe de travail Seine sont moins enthousiastes sur les possibilités du réseau commuté actuel. Il faut tenir compte des appels qui n'aboutissent pas, des problèmes d'encombrement de réseau; de la fragilité du fil et des travaux de génie civil qu'il impose. La qualité du réseau est encore très en retard par rapport à ce qu'elle est en Belgique ou en Allemagne, ne serait-ce qu'en ce qui concerne les capacités de transmission des lignes.

M. PERSOONS. - Le réseau du Service "Etudes Hychologiques" des Travaux Publics, lorsqu'il comportera 100 postes connectés, aura coûté environ 1,2 millions de francs.

Les balises ARGOS coûtent $50000 \mathrm{~F}$ chacune.

M. BEDIOT. -20000 ! Et, il faut prendre en compte le fait qu'elles ne nécessitent aucun génie civil.

M. PERSOONS. - Les prix chutent à une vitesse extraordinaire.

M. GUILLOT. - En ce qui concerne ARGOS, je pense qu'il faut réserver ce moyen aux emplacements non accessibles au réseau auto-commuté. C'est-à-dire aux pays en voie de développement, aux sites de montagne, et ne pas encombrer ce réseau avec des balises inutiles. La capacité du satellite est de 16000 balises pour le monde entier. Plusieurs centaines de balises dans une petite région comme la France vont saturer le satellite et créer des pertes. Encore une fois il vaut mieux utiliser le réseau public là où c’est possible.

M. BEDIOT. - En fait, la saturation ne commencerait que pour environ 900 balises dans un rayon de $1500 \mathrm{~km}$ environ. Les besoins actuels en Europe Occidentale sont inférieurs à la centaine. Le pire serait de laisser ce système inexploité.

D'autre part, nous ne pensons pas qu'ARGOS soit la meilleure solution, c'est uniquement la moins chère actuellement. Nous envisageons le recours à METEOSAT.

M. PERSOONS. - La pérennité de METEOSAT n'est pas assurée. D'autre part, il est en éclipse 2 fois 24 heures par an pour des questions de piles solaires. Actuellement le canal "télédétection" ne fonctionne plus.

M. BEDIOT. - Il est prévu deux satellites en permanence se relayant. C'est la solution de l'avenir.

Le Président. - Je remercie tous ceux qui ont participé à cette discussion.

La Météorologie Nationalc a mis en place, dans le Sud-Est de la France, un réseau "incendies de forêts" avec l'aide de la Direction de la Sécurité Civile. Le réseau radio adopté permet de transmettre à partir de sites inaccessibles au réseau-commuté 
C'est onéreux, et je crois qu'il faut, chaque fois que c'est possible, utiliser les équipements nationaux, communs, qui, mieux rentabilisés, seront l'objet d'amélioration de fiabilité

Pour les sites isolés, les satellites ont un rôle important à jouer. En ce qui conerne METEOSAT, le prochain satellite doit être lancé par la fusée ARIANE le 19 juin. Pour le satellite suivant, les discussions sont en cours pour la répartition des charges financières au niveau européen. Ainsi la fiabilité à long terme de ce mode de transmission n'est pas complètement assurée.

\section{Abstract \\ Hydrological telemeasurement using a public telephone system}

In Belgium, the public telephone system is used for transmission of analogical facts. This telemeasurement system, developped by the Agricultural Engineering Department of the Catholic University of Louvain, is presently operational in four administrations, among which the hydrological Study Service of the Ministry of Public Works. The system is composed of automatic receiving machines which, on receipt of a call, transmit on the telephone line analogical information (pure frequencies of $600-800 \mathrm{~Hz}$ ) corresponding either to measurements at the time of the call or to measurements reflecting the history of a phenomenon (last 10 timed values). These receivers require no local power. At the call centre, a central set, managed by a microprocessor, enables calls to be sent out (automatically or manually); can receive and measure the analogical data received from the automatic receivers; and process this data in order to transform it into directly useable values and transfer it all to a mass memory.
The receivers are compatible with all analogical or digital receivers. The information is returned to a precision of around $0.1 \%$.

To date, 8 microprocessor calling sets and more than 200 field sets are in use on a permanent basis in Belgium.

More specifically, the Hydrological Study Service has equipped its measurement network with 65 telelimnimetres and 15 pluviographs. Thanks to this network, the HSS was able to follow, in actual time, the exceptional increase in the level of the waters of the Meuse on 20th and 21st July 1980.

The availability in real time of the level and rainfall measurements enabled the duty officer to foresee the evolution of the rising level and to take the necessary precautions. During this difficult and critical time, the percentage of accurate data received by the $H S S$ was $92 \%$ and this in spite of the fact that this was in the face of disaster. 